Reprinted from Physiological Plant Pathology (1977) 11, 287-296

Characterization of components from culture filtrates of Botrytis cinerea which stimulate phaseollin biosynthesis in Phaseolus vulgaris cell suspension cultures

R. A. Dixon and K. W. Fuller

Botany School, South Parks Road, Oxford OXI 3RA, U.K. 


\title{
Characterization of components from culture filtrates of Botrytis cinerea which stimulate phaseollin biosynthesis in Phaseolus vulgaris cell suspension cultures
}

\author{
R. A. Dixon $\dagger$ and K. W. Fuller \\ Botany School, South Parks Road, Oxford OX1 3RA, U.K. \\ (Accepted for publication June 1977)
}

\begin{abstract}
Sterile culture filtrates from shake cultures of Botrytis cinerea stimulated phaseollin biosynthesis in cell suspension cultures of Phaseolus vulgaris. Increased phaseollin levels in induced cultures were observed after about $12 \mathrm{~h}$. The inducing activity in the filtrate was heat stable and soluble in $80 \%$ ethanol. Gel-filtration studies showed several peaks of inducing activity, and pronase digestion destroyed the activity of the two highest molecular weight peaks. Treatment of culture filtrates with ribonuclease or deoxyribonuclease did not remove inducing activity and even after inactivation by autoclaving these enzymes were potent inducers of the phytoalexin response.
\end{abstract}

\section{INTRODUCTION}

It is now believed that phytoalexin production in several natural plant-fungus interactions is stimulated in the host cells by compounds of fungal origin termed "inducers" or elicitors. Fungal culture filtrates or mycelial extracts have been shown to induce necrosis and/or phytoalexin production in a number of plant species $[6,23,25,27]$ and the use of these may approximate more to the normal parasite-host relationship than the use of the heavy metal ions or antibiotics which have been much used as inducers of the phytoalexin response in previous studies [9, 19, 22, 24, 25]. Recently, several phytoalexin-inducing substances from fungi have been separated and characterized. These include a high molecular weight polysaccharide fraction from culture filtrates and mycelial walls of Colletotrichum lindemuthianum [2] and fractions (largely carbohydrate) from culture filtrates and mycelial walls of Phytophthora megasperma var. sojae [3-5]. Monilicolin A, a low molecular weight polypeptide from mycelium of Monilinia fructicola (a pathogen of stone fruit), induces the biosynthesis of phaseollin in bean pods, but not of pisatin in peas [8], and pisatin-inducing components from culture filtrates of Fusarium solani also appear to be, at least in part, proteinaceous [10]. In a few of these cases the possible rôle of the inducers in imparting specificity to plant-parasite interactions has been investigated $[5,10,18]$.

Cell suspension cultures of Phaseolus vulgaris have been shown to produce the phytoalexin phaseollin in the absence of fungal or chemical stimulation, and the extent of this non-induced production is affected by the levels of plant growth regulators in the culture medium [12]. In cell suspensions of soybean synthesis of the

$\dagger$ Present address: Department of Biochemistry, Tennis Court Road, University of Cambridge, Cambridge CB2 1QW, U.K. 
phytoalexin glyceollin could be induced by treatment with the elicitor from $P$. megasperma var. sojae [13]. In the present paper we report the preliminary characterization of components from culture filtrates of Botrytis cinerea capable of inducing phaseollin biosynthesis in cell suspension cultures of $P$. vulgaris. $B$. cinerea, which shows less specialization than most fungi with respect to host range, has been shown to induce phaseollin biosynthesis in excised bean pods [9].

\section{MATERIALS AND METHODS}

\section{Plant tissue cultures}

The tissue culture medium used in this work was a modification of the medium of Schenk \& Hildebrandt [26] (medium B) and has been described elsewhere [12]. Medium B contains, in addition to salts, organic supplement and sucrose, the growth regulators $p$-chlorophenoxyacetic acid $\left(10^{-5} \mathrm{M}\right)$, 2,4-dichlorophenoxyacetic acid $\left(2 \times 10^{-6} \mathrm{M}\right)$ and kinetin $\left(5 \times 10^{-7} \mathrm{M}\right)$.

Stock callus cultures were grown on medium B (semi-solid). Callus lumps (approximately $8 \mathrm{~g}$ total fresh weight) were used as inocula for flasks of liquid medium $\mathrm{B}(250 \mathrm{ml})$, and pipettable suspensions were obtained on agitation. All cultures were grown at $25^{\circ} \mathrm{C}$ on reciprocal shakers $\left(1 \frac{1}{2}\right.$ in throw, 150 throws min $\left.^{-1}\right)$ under a photorégime of $12 \mathrm{~h}$ light/12 h dark in a room lit by a single $80 \mathrm{~W}$ (natural) fluorescent tube approximately $2 \mathrm{~m}$ from the cultures, and were subcultured at 5 week intervals by transferring $30 \mathrm{ml}$ inocula to $220 \mathrm{ml}$ batches of fresh medium. Suspensions 21 days after subculture were used in all the experiments described in this paper.

\section{Measurement of fresh weight}

Fresh weights of suspension-cultured cells were measured by weighing after centrifugation of suitable aliquots of suspension through pre-weighed sintered glass centrifuge filters.

\section{Preparation of fungal culture filtrates}

Stock cultures of Botrytis cinerea (obtained from the Commonwealth Mycological Institute, Kew, Surrey) were maintained as slopes on modified Czapek-Dox agar (Oxoid Ltd). For preparation of culture filtrates, 11 conical flasks containing $300 \mathrm{ml}$ of Czapek-Dox liquid medium were inoculated with conidia of the fungus. Incubation was for 14 days in the dark at $25^{\circ} \mathrm{C}$ on an orbital shaker (150 rev/min). After this time the cultures were harvested and the mycelium removed by centrifugation at $8000 \mathrm{~g}$ for $1.5 \mathrm{~h}$. The culture filtrate above the mycelial pellet was pipetted off, diluted $1: 1$ with distilled water and stored at $-15^{\circ} \mathrm{C}$ until required.

\section{Preparation of ethanol-soluble and ethanol-insoluble fractions}

Four hundred millilitres of absolute ethanol were added slowly and with stirring to $100 \mathrm{ml}$ of culture filtrate. After standing for $30 \mathrm{~min}$, the precipitate that formed was centrifuged at $25000 \mathrm{~g}$ for $30 \mathrm{~min}$. The clear supernatant was taken to dryness under reduced pressure at $40^{\circ} \mathrm{C}$, and the residue taken up in $100 \mathrm{ml}$ of distilled water. This fraction is referred to as the $80 \%$ ethanol-soluble fraction of the culture filtrate. The 
pellet (ethanol-insoluble material) was washed twice with $80 \%$ ethanol and taken up in $100 \mathrm{ml}$ of distilled water.

\section{Gel-filtration}

For application to Sephadex gel-filtration columns, $100 \mathrm{ml}$ of $80 \%$ ethanol-soluble fraction were taken to dryness under reduced pressure at $40^{\circ} \mathrm{C}$ and the residue dissolved in $5 \mathrm{ml}$ of $10 \mathrm{~mm}$ phosphate-citrate buffer, $\mathrm{pH} \mathrm{6.2.} \mathrm{Columns} \mathrm{of} \mathrm{Sephadex}$ G-50 $(32 \times 2.5 \mathrm{~cm})$ and G-25 $(42 \times 2 \mathrm{~cm})$ were equilibrated with this buffer which was also used as eluant.

\section{Determination of phaseollin}

Phaseollin was determined by an extension of the method of Cruickshank \& Perrin [9]. The ultraviolet absorption of the compound was measured after separation by thin-layer chromatography on silica plates with toluene : ethyl formate : formic acid $(7: 2: 1)$ as solvent, as described previously [12].

\section{Measurement of phaseollin induction by radioisotope incorporation}

Replicate $8 \mathrm{ml}$ aliquots of suspension culture were aseptically transferred to $25 \mathrm{ml}$ conical flasks containing $2 \mathrm{ml}$ of sterile complete or fractionated culture filtrate or, in controls, $10 \mathrm{~mm}$ phosphate-citrate buffer or Czapek-Dox medium (the latter diluted $1: 1$ with distilled water). Flasks were reciprocally shaken ( 150 throws $\left.\mathrm{min}^{-1}\right)$ for $16 \mathrm{~h}$ at $25^{\circ} \mathrm{C}$ prior to the addition of $0.1 \mu \mathrm{mol}(0.5 \mu \mathrm{Ci}) \mathrm{L}-\left[\mathrm{U}^{14} \mathrm{C}\right]$ phenylalanine per flask. The radioactive phenylalanine was purchased ready sterilized (Radiochemical Centre, Amersham, U.K.). Unlabelled phenylalanine for dilution of the precursor to the required specific activity was sterilized by passing through a $0.2 \mu \mathrm{m}$ pore-size membrane filter. Flasks were incubated for a further $60 \mathrm{~h}$ after isotope addition. Cells were then harvested by centrifuging at $1200 \mathrm{~g}$ for $5 \mathrm{~min}$. Supernatant medium was decanted and the cells washed twice with $5 \mathrm{ml}$ of culture medium containing $0.1 \mathrm{~mm}$ unlabelled phenylalanine before extraction and separation of phaseollin as previously described [12]. Phaseollin bands $\left(R_{\mathrm{F}} 0.56\right)$ on thin-layer chromatograms were cut out and counted for radioactivity in a Tracerlab Spectro/matic liquid scintillation counter using a scintillation fluid consisting of $5 \mathrm{~g}$ of butylphenyl-biphenyl oxadiazole per 1 of Analar toluene (scintillant A, counting efficiency $90 \%$ ). For determination of radioactivity in the phaseollin in ethanolic eluates after ultraviolet spectrophotometry, $0.5 \mathrm{ml}$ of the solution was counted in $3.5 \mathrm{ml}$ of scintillation fluid consisting of $7 \mathrm{~g}$ of butylphenyl-biphenyl oxadiazole and $80 \mathrm{~g}$ of naphthalene in $600 \mathrm{ml}$ of Analar toluene plus $600 \mathrm{ml}$ of Analar 2-methoxyethanol (scintillant B, counting efficiency $90 \%$ ). Results are expressed as ct $/ \mathrm{min}$ in the extracted phaseollin from two pooled replicate induction flasks.

The two "unlabelled phenylalanine washes" from the cell samples were pooled with their respective initial supernatants and $200 \mu l$ samples counted in $8 \mathrm{ml}$ of scintillant B for estimation of the \% uptake of radioisotope, which was between 75 and $85 \%$ in all the experiments described.

Gas-liquid chromatography of a trimethylsilyl-derived ethanolic eluate of the material at $R_{\mathrm{F}} 0.56$ on thin-layer chromatograms showed the presence of no 
compounds other than phaseollin. Radioactivity incorporated into phaseollin under the conditions described was proportional to the amount of phaseollin produced in the flasks per unit fresh weight of tissue (as shown in the last columns of Tables 1 and 2 ). The percentage of the total radioactivity applied that became incorporated into phaseollin was between 0.02 (lowest control value) and $0.59 \%$ (highest induced value).

\section{Determination of protein}

(a) Unfractionated culture filtrates. To $3 \mathrm{ml}$ samples, an equal volume of $10 \%$ trichloroacetic acid was added and the protein content of the precipitates determined by the method of Lowry et al. as modified by Leggett-Bailey [20].

(b) Fractions from gel-filtration. Suitable aliquots were made up to $1.5 \mathrm{ml}$ with $0.2 \mathrm{M}$ sodium borate $\mathrm{pH} 9.3$, and $0.3 \mathrm{ml}$ of a solution of $15 \mathrm{mg}$ fluorescamine (4-phenylspiro[furan-2(3), 1'-phthalan]3,3-dione) per $100 \mathrm{ml}$ Analar acetone [28] was added rapidly to each tube whilst shaking on a Vortex mixer. The fluorescence of the samples was read on a Baird Atomic Fluorimet FM 200 fluorimeter, excitation filter No. OB10 (transmission maximum $425 \mathrm{~nm}$ ), emission filter No. OY13 (transmission maximum $550 \mathrm{~nm}$ ). A suitable range of $\mathrm{D}, \mathrm{L}$-alanine concentrations and a reagent blank were used to standardize the instrument, and all results are expressed as nmol equivalent $\mathrm{D}, \mathrm{L}$-alanine by this assay method.

\section{Determination of total carbohydrate}

The total carbohydrate content of fractions from gel-filtration columns was determined by the method of Fuller \& Northcote [14] by reaction with $0.4 \%$ 1-naphthol in concentrated sulphuric acid. D-Glucose was used as standard, and all results are expressed as nmol equivalent glucose.

\section{RESULTS}

Response of suspension-cultured cells to unfractionated culture filtrate

Suspension-cultured cells of Phaseolus vulgaris, 21 days after transfer to fresh medium $B$, produced phaseollin in response to treatment with sterile culture filtrates of Botrytis cinerea (Fig. 1). Increased levels of the phytoalexin were first detected in the cultures after 12 to $18 \mathrm{~h}$. As previous work had indicated [12], phaseollin production occurred to a lesser but nevertheless measurable extent in the absence of culture filtrate. In this paper the term "induction" is, therefore, used to describe the stimulation of phaseollin biosynthesis above the control rates.

\section{Heat stability of the inducing activity}

The ability of the culture filtrate to stimulate phaseollin biosynthesis in the cultures was the same whether sterilization was by autoclaving at $120^{\circ} \mathrm{C}$ for $20 \mathrm{~min}$ or by microfiltration through $0.2 \mu \mathrm{m}$ pore size Millipore membrane filters. In view of the difficulty of microfiltration of relatively viscous solutions, culture filtrates were sterilized by autoclaving in all the experiments here described. 


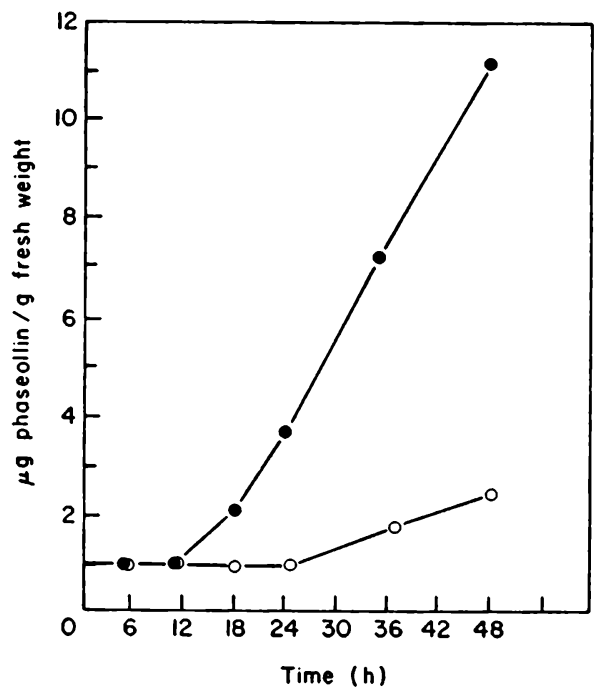

Fig. 1. Production of phaseollin by suspension cultures of $P$. vulgaris in response to a sterile

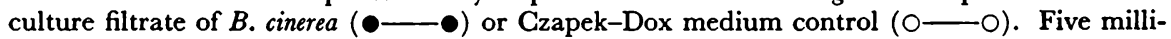
litre aliquots of inducing or control solution were added to $20 \mathrm{ml}$ volumes of $P$. vulgaris suspension in $50 \mathrm{ml}$ conical flasks. Phaseollin was extracted and determined spectrophotometrically as described.

Ethanol fractionation and enzymic treatment of the culture filtrate

The filtrate contained ethanol-insoluble polysaccharide material (accounting for about $5 \%$ of the total carbohydrate content of the filtrate). However, the results in Table 1 indicate that all the phaseollin-inducing activity was $80 \%$ ethanol-soluble.

TABLE 1

Eighty per cent ethanol fractionation of the phaseollin-inducing activity in a B. cinerea culture filtrate ${ }^{a}$

\begin{tabular}{|c|c|c|c|}
\hline Inducing solution & $\begin{array}{l}\text { Phaseollin produced } \\
(\mu \mathrm{g}) / \mathrm{g} \text { fresh weight }\end{array}$ & ct/min in phaseollin & $\frac{\mathrm{ct} / \mathrm{min} \text { in phaseollin }}{\text { phaseollin }(\mu \mathrm{g}) / \mathrm{g} \text { fresh weight }}$ \\
\hline Unfractionated culture filtrate & $10 \cdot 00$ & 3847 & $384 \cdot 7$ \\
\hline $80 \%$ ethanol-insoluble fraction & $1 \cdot 19$ & 445 & $371 \cdot 7$ \\
\hline $80 \%$ ethanol-soluble fraction & $10 \cdot 60$ & 3875 & $365 \cdot 6$ \\
\hline Reconstituted filtrate & $9 \cdot 30$ & 3641 & $391 \cdot 5$ \\
\hline Distilled water & 0.96 & 356 & $370 \cdot 8$ \\
\hline
\end{tabular}

a The amount of phaseollin in the cells at zero time was $2 \mu \mathrm{g} / \mathrm{g}$ fresh weight.

Samples of the ethanol-soluble fraction of the culture filtrate ( $\mathrm{pH}$ adjusted to 6.0) were treated with pronase, ribonuclease or deoxyribonuclease and the solutions tested for phaseollin-inducing activity as shown in Table 2. Pronase treatment reduced the inducing activity of the filtrate by about $30 \%$. Ribonuclease and deoxyribonuclease, although both enzymically inactive as a result of autoclaving, were potent stimulators of phaseollin biosynthesis. However, culture filtrate treated with these two enzymes gave higher induction values than the corresponding controls, 
TABLE 2

Effect of enzyme treatments on the phaseollin-inducing activity of the $80 \%$ ethanol-soluble fraction of $a$ B. cinerea culture filtrate ${ }^{a}$

\begin{tabular}{|c|c|c|c|c|}
\hline $\begin{array}{l}\text { Inducing } \\
\text { solution }\end{array}$ & Treatment & $\begin{array}{l}\text { Phaseollin produced } \\
(\mu \mathrm{g}) / \mathrm{g} \text { fresh weight }\end{array}$ & $\mathrm{ct} / \mathrm{min}$ in phaseollin & $\frac{c t / \min \text { in phaseollin }}{\text { phaseollin }(\mu \mathrm{g}) / \mathrm{g} \text { fresh weight }}$ \\
\hline $\begin{array}{l}\text { Ethanol-soluble } \\
\text { fraction }\end{array}$ & $\begin{array}{l}\text { None } \\
\text { Pronase } \\
\text { RNAase } \\
\text { DNAase }\end{array}$ & $\begin{array}{r}12 \cdot 84 \\
9 \cdot 50 \\
34 \cdot 00 \\
34 \cdot 20\end{array}$ & $\begin{array}{r}4800 \\
3615 \\
11658 \\
11480\end{array}$ & $\begin{array}{l}373 \cdot 8 \\
380 \cdot 5 \\
342 \cdot 8 \\
335 \cdot 7\end{array}$ \\
\hline Distilled water & $\begin{array}{l}\text { None } \\
\text { Pronase } \\
\text { RNAase } \\
\text { DNAase }\end{array}$ & $\begin{array}{r}3 \cdot 36 \\
3 \cdot 93 \\
25 \cdot 35 \\
20 \cdot 30\end{array}$ & $\begin{array}{l}1186 \\
1478 \\
8817 \\
7225\end{array}$ & $\begin{array}{l}353 \cdot 0 \\
376 \cdot 1 \\
347 \cdot 8 \\
355.9\end{array}$ \\
\hline
\end{tabular}

a Two millilitre samples of the ethanol-soluble fraction ( $\mathrm{pH}$ adjusted to $6 \cdot 0$ ) were treated with $1 \mathrm{mg}$ of either pronase, ribonuclease or deoxyribonuclease (obtained from B.D.H. Ltd, U.K.). Control solutions had distilled water in place of the filtrate fraction. After incubation at $40^{\circ} \mathrm{C}$ (pronase) or $25^{\circ} \mathrm{C}$ (RNAase and DNAase) for $4 \mathrm{~h}$, the solutions were autoclaved to sterilize. When cool, $8 \mathrm{ml}$ aliquots of $P$. vulgaris cell suspension were added to each $2 \mathrm{ml}$ of treated inducer or control solution, and radioisotope incorporation was measured as described in Materials and Methods.

suggesting that RNA and DNA were not components of the phaseollin-inducing activity.

\section{Gel-filtration studies}

Two major peaks of phaseollin-inducing activity were obtained on gel-filtration of the ethanol-soluble fraction of the culture filtrate on Sephadex G-50 (Fig. 2). The $V_{\mathrm{e}} / V_{\mathrm{o}}$ value for the higher molecular weight peak was slightly greater than that for a sample of cytochrome $c$ (mol. wt 12 270) applied to the same column. The major (lower molecular weight) peak was associated with the bulk of the carbohydrate and

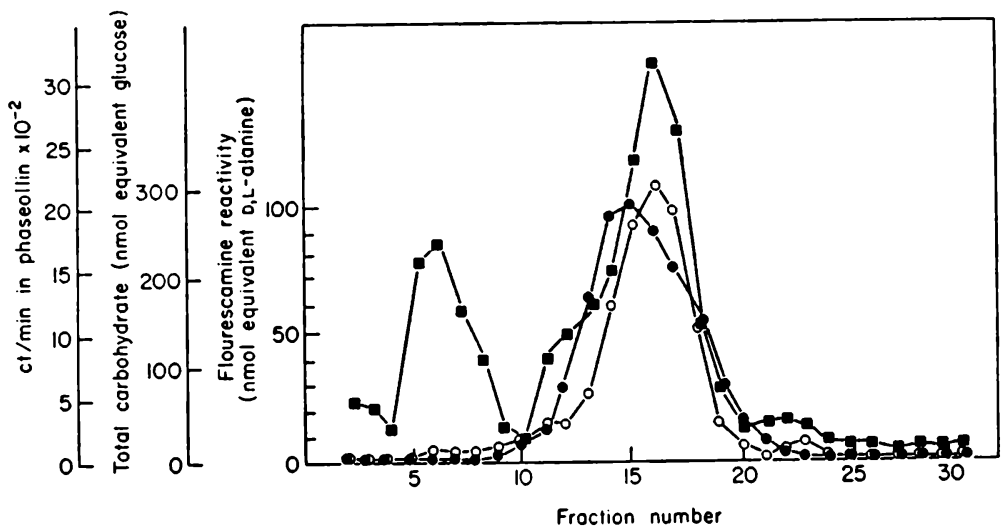

Fig. 2. Sephadex G-50 gel-filtration of the $80 \%$ ethanol-soluble fraction prepared from an original $100 \mathrm{ml}$ of $B$. cinerea culture filtrate. The fraction size was $5 \mathrm{ml}, 10 \mathrm{ml}$ was run through the column before fractions were collected and the flow rate was $60 \mathrm{ml} / \mathrm{h}$. Profiles are shown for phaseollin-inducing activity ( $\square-\square)$, total carbohydrate $(\mathrm{O}-\mathrm{O})$ and fluorescamine reactivity (-๑). 


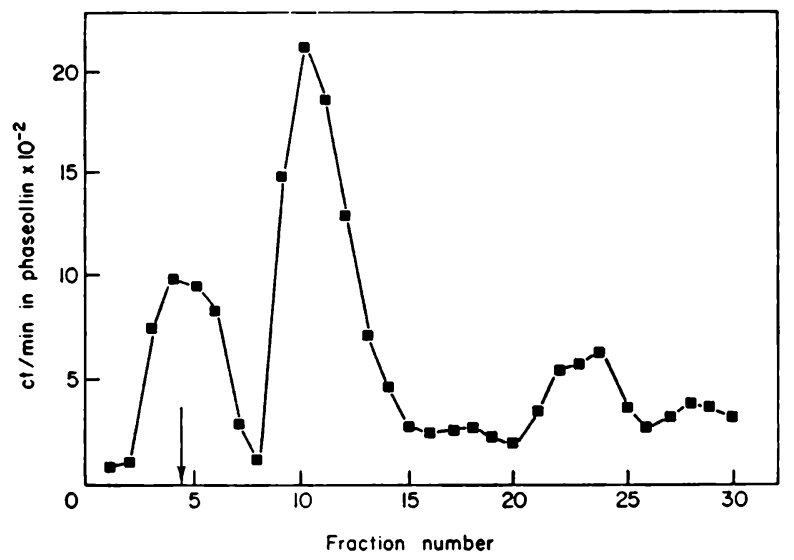

Fig. 3. Sephadex G-25 elution profile of the phaseollin-inducing activity in a concentrate of fractions 13 to 19 from the G-50 separation shown in Fig. 2. The fraction size was $6.2 \mathrm{ml}$, $35 \mathrm{ml}$ was run through the column before fractions were collected and the flow rate was $60 \mathrm{ml} / \mathrm{h}$. The arrow indicates the void volume of the column, as determined by the exclusion of blue dextran (mol. wt $\left.2 \times 10^{6}\right)$.

fluorescamine-reacting material eluting from the column. Protein was not detected in the unfractionated culture filtrate by the method of Leggett-Bailey.

Fractions 13 to 19 (Fig. 2) were pooled, reduced to $5 \mathrm{ml}$ under vacuum and re-cycled through Sephadex G-25 (Fig. 3). Three phaseollin-inducing peaks were resolved by this method. G-25 gel-filtration of the ethanol-soluble fraction of the filtrate after incubation with pronase (Fig. 4) showed the loss of the higher molecular weight inducing fractions (those emerging from the columns first in Figs 2 and 3). It is therefore likely that the reduction in inducing activity as a result of pronase

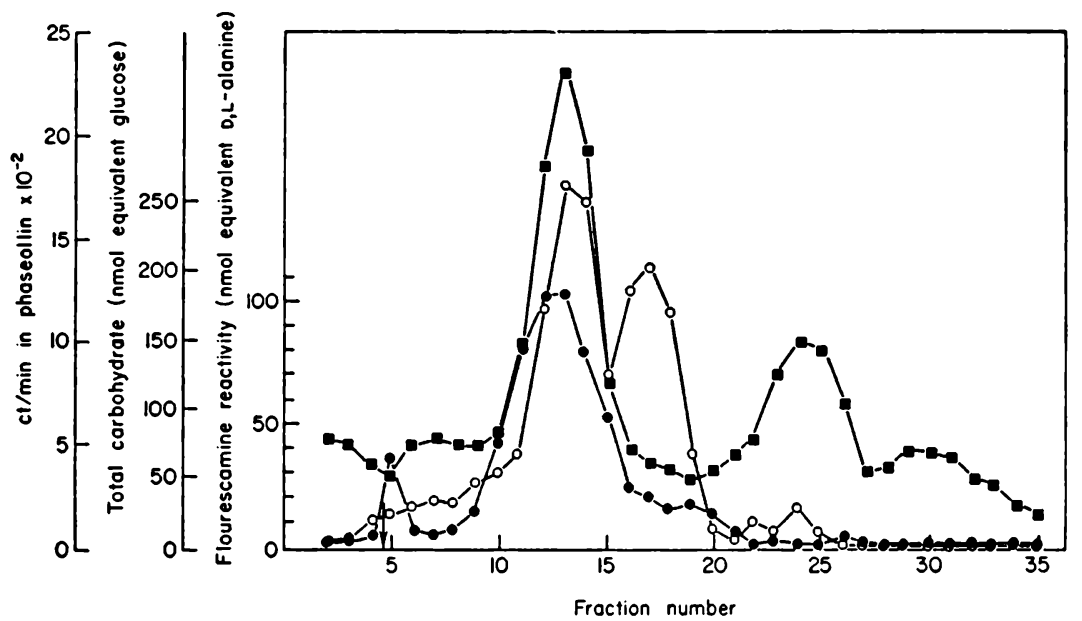

Fig. 4. Sephadex G-25 gel-filtration of the $80 \%$ ethanol-soluble fraction of a $B$. cinerea culture filtrate after pronase treatment. Details as in the legend to Fig. 3. Profiles are shown for phaseollin-inducing activity ( $\square-\square)$, total carbohydrate $(\mathrm{O}-\mathrm{O})$ and fluorescamine reactivity (-๑). 
treatment (Table 2) is a consequence of the loss of activity of these fractions. The other inducing peaks were unaffected by pronase treatment. As with the G-50 fractionation, the majority of the fluorescamine-reacting material and about half of the total carbohydrate eluting from the G-25 column was associated with the major inducing peak.

\section{DISCUSSION}

The results presented in this paper indicate that sterile, cell-free culture filtrates of Botrytis cinerea contain components which are active as inducers of increased phaseollin production in cell suspension cultures of Phaseolus vulgaris. B. cinerea is an unspecialized parasite, attacking a wide variety of fruits, vegetables and ornamentals. There are several reports of its ability to induce resistance responses in host plants. As early as 1927 it was reported that treatment of seeds of $P$. vulgaris with culture medium that had supported growth of the fungus led to increased resistance of the seedlings which developed subsequently [30]. Cruickshank \& Perrin [9] have described the interaction between two different isolates of the fungus with the French bean cultivar Red Kidney. Although one isolate caused a susceptible and the other a resistant interaction, these outcomes were not ascribed to differences in the amounts of phaseollin produced during infection, but rather to differences in the median effective dose ranges of phaseollin for inhibition of spore germination of the two isolates. The results of Deverall \& Vessey [11] and Letcher et al. [21] demonstrate that the acetylenic phytoalexins wyerone and wyerone acid are induced in the broad bean in response to infection by Botrytis. These reports formed the basis for the present attempt to demonstrate inducers of phytoalexin biosynthesis in culture filtrates from the fungus.

The lag of about $12 \mathrm{~h}$ before the appearance of induced phaseollin in the cell suspensions is of a similar time scale to that observed in bean tissues when treated with a wide variety of inducing agents $[9,25]$. However, the extent of phaseollin production observed here after treatment with unfractionated culture filtrate was lower than occurs in tissues of the whole plant responding to fungal infection [7] or chemical inducers [17], although results to be reported in a later paper indicate that rates of induced production can be greatly increased by altering the chemical composition of the culture medium. The medium B used in the present work was chosen because of its ability to support good growth of the suspensions, and because of the low background levels of phaseollin production associated with this growth.

In view of their solubility in $80 \%$ ethanol, the $B$. cinerea fractions which induce phaseollin do not appear to be high molecular weight polysaccharides like the inducer from Colletotrichum lindemuthianum [2], and the pronase digestion studies suggest that protein is a component of the two inducing fractions of higher molecular weight. The low carbohydrate and peptide content of these fractions suggests that the components are active at very low concentrations. The other inducing fractions are of much lower molecular weight, and in this respect at least may resemble the racespecific elicitor isolated by Keen from culture filtrates of Phytophthora megasperma var. sojae [18].

Both ribonuclease and deoxyribonuclease were effective inducers of phaseollin biosynthesis in the bean cultures, and the effects of these proteins plus culture filtrate 
were additive. Hadwiger and his associates $[15,16]$ have proposed that ribonuclease and other polypeptides, such as poly-L-lysine and poly-L-arginine, induce pisatin production in pea pods as a result of the net basic nature of their polypeptide backbones, which might enable them to bind to specific regions of chromatin and switch on the expression of the genes for the phytoalexin pathway. It is not clear, however, whether denatured ribonuclease is able to enter the cells. Furthermore, basic polypeptides such as poly-L-lysine can bring about altered metabolism in animal cells by binding at the plasma membrane [29]. A similar concept involving receptor sites in the plasma membrane of the plant cell, which can recognize fungal cell wall components, has been proposed as a model for race-specific resistance interactions between plants and their fungal pathogens [1]. Moreover, the work of Ayers et al. [3-5] is strongly suggestive of a mechanism for phytoalexin induction involving cell surface recognition phenomena, although it is still not resolved whether this recognition is itself the major determinant of race specificity. The present $B$. cinerea-bean culture system could be useful for studying the mode of action of inducers at the cellular level, as well as being a convenient system for their assay and for the study of isoflavonoid biosynthesis in general.

The authors thank Mrs D. Marsden for technical assistance. R. A. Dixon was in receipt of a Science Research Council studentship during the course of the work.

\section{REFERENCES}

1. Albersheim, P. \& Anderson-Prouty, A. J. (1975). Carbohydrates, proteins, cell surfaces and the biochemistry of pathogenesis. Annual Review of Plant Physiology 26, 31-52.

2. Anderson-Prouty, A. J. \& Albersheim, P. (1975). Host-pathogen interactions. VIII. Isolation of a pathogen-synthesized glucan that elicits a defence response in the pathogen's host. Plant Physiology 56, 286-291.

3. Ayers, A. R., Ebel, J., Finelli, F., Berger, N. \& Albersheim, P. (1976). Host-pathogen interactions. IX. Quantitative assays of elicitor activity and characterization of the elicitor present in the extracellular medium of cultures of Phytophthora megasperma var. sojae. Plant Physiology 57, 751-759.

4. Ayers, A. R., Ebel, J., Valent, B. \& Albersheim, P. (1976). Host-parasite interactions. X. Fractionation and biological activity of an elicitor isolated from the mycelial walls of Phytophthora megasperma var. sojae. Plant Physiology 57, 760-765.

5. Ayers, A. R., Valent, B., Ebel, J. \& Albersheim, P. (1976). Host-pathogen interactions. XI. Composition and structure of wall-released elicitor fractions. Plant Physiology 57, 766-774.

6. Bailey, J. A. (1969). Effects of antimetabolites on the production of the phytoalexin pisatin. Phytochemistry 8, 1393-1395.

7. Bailey, J. A. (1973). Phaseollin accumulation in Phaseolus rulgaris following infection by fungi, bacteria and a virus. In Fungal Pathogenicity and the Plant's Response, Ed. by R. J. W. Byrde \& C. V. Cutting. Academic Press, London.

8. Cruickshank, I. A. M. \& Perrin, D. R. (1968). The isolation and partial characterisation of monilicolin A, a polypeptide with phaseollin inducing activity from Mlonilinia fructicola. Life Sciences 7, 449-458.

9. Cruickshank, I. A. M. \& Perrin, D. R. (1971). Studies on phytoalexins. XI. The induction, antimicrobial spectrum and chemical assay of phaseollin. Phytopathologische Zeitschrift 70, 209229.

10. Daniels, D. L. \& Hadwiger, L. A. (1976). Pisatin-inducing components in filtrates of virulent and avirulent Fusarium solani cultures. Physiological Plant Pathology 8, 9-19.

11. Deverall, B. J. \& Vessey, J. C. (1969). Role of a phytoalexin in controlling lesion development in leaves of Vicia faba after infection by Botrytis spp. Annals of Applied Biology 63, 449-458.

12. Dixon, R. A. \& F cller, K. W. (1976). Effects of synthetic auxin levels on phaseollin production and phenylalanine ammonia-lyase (PAL) activity in tissue cultures of Phaseolus rulgaris L. Physiological Plant Pathology 9, 299-312. 
13. Ebel, J., Ayers, A. R. \& Albersheim, P. (1976). Host-pathogen interactions. XII. Response of suspension cultured soybean cells to the elicitor isolated from Phytophthora megasperma var. sojae, a fungal pathogen of soybeans. Plant Physiology 57, 775-779.

14. Fuller, K. W. \& Northcote, D. H. (1956). A micro-method for the separation and determination of polysaccharides by zone electrophoresis. Biochemical Journal 64, 657-663.

15. Hadwiger, L. A. \& Schwochau, M. E. (1970). Induction of phenylalanine ammonia-lyase and pisatin in pea pods with polylysine, spermidine or histone fraction. Biochemical and Biophysical Research Communications 38, 683-688.

16. Hadwiger, L. A., Jafri, A., von Broembsen, S. \& Eddy, R. (1974). Mode of pisatin induction. Increased template activity and dye binding capacity of chromatin isolated from polypeptidetreated pea pods. Plant Pathology 53, 52-63.

17. Hess, S. L. \& Hadwiger, L. A. (1971). The induction of phenylalanine ammonia-lyase and phaseollin by 9 -aminoacridine and other DNA-intercalating compounds. Plant Physiology 48, 197-202.

18. KEEN, N. T. (1974). Specific elicitors of phytoalexin production: determinants of race specificity in pathogens. Science 187, 74-75.

19. KEEN, N. T., ZAKI, A. I. \& Sims, J. J. (1972). Biosynthesis of hydroxyphaseollin and related isoflavonoids in disease-resistant soybean hypocotyls. Phytochemistry 11, 1031-1039.

20. Leggett-Bailey, J. (1962). In Techniques in Protein Chemistry, Chapter 11. Elsevier Publishing Co., Amsterdam.

21. Letcher, R., Widdowson, D. A., Deverall, B. J. \& Mansfield, J. W. (1970). Identification and activity of wyerone acid as a phytoalexin in broad bean (Vicia faba) after infection by Botrytis. Phytochemistry 9, 249-252.

22. Munn, C. B. \& Drysdale, R. B. (1975). Kievitone production and phenylalanine ammonia-lyase activity in cowpea. Phytochemistry 14, 1303-1307.

23. Neusch, J. (1963). Defence reactions in orchid bulbs. Society of General Microbiology Symposium, No. 13, pp. 335-343.

24. Perrin, D. R. \& Cruickshank, I. A. M. (1965)). Studies on phytoalexins. VII. Chemical stimulation of pisatin formation in Pisum sativum L. Australian Journal of Biological Sciences 18, 803816.

25. Rathmell, W. G. \& Bendall, D. S. (1971). Phenolic compounds in relation to phytoalexin biosynthesis in hypocotyls or Phaseolus vulgaris. Physiological Plant Pathology 1, 351-362.

26. Schenk, R. H. \& Hildebrandt, A. C. (1971). Medium and techniques for induction and growth of monocotyledonous and dicotyledonous plant cell cultures. Canadian Journal of Botany 50, 199-204.

27. Tомryama, K. (1971). Cytological and biochemical studies on the hypersensitive reaction of potato cells to Phytophthora infestans. In Morphological and Biochemical Events in Plant-Parasite Interactions, Ed. by S. Akai \& S. Ouchi. The Phytopathological Society of Japan, Tokyo.

28. Udenfreid, S., Stein, S., Bohlen, P., Dairman, W., Leimgruber, W. \& Weigele, M. (1972). Fluorescamine; a reagent for assay of amino acids, peptides, proteins and primary amines in the picomole range. Science 178, 871-872.

29. Whitfield, J. F., Perris, A. D. \& Youdale, T. (1968). The rôle of calcium in the mitotic stimulation of rat thymocytes by detergents, agmatine and poly-L-lysine. Experimental Cell Research 53, 155-165.

30. Wood, R. K. S. (1967). Physiological Plant Pathology, p. 507. Blackwell Scientific Publications, Oxford. 
